\title{
Cost Effectiveness of Mirabegron Compared with Antimuscarinic Agents for the Treatment of Adults with Overactive Bladder in Colombia
}

\author{
Hélène Parise ${ }^{1} \cdot$ Robert Espinosa $^{1}\left[\right.$ [ $\cdot$ Katherine Dea ${ }^{1} \cdot$ Pablo Anaya $^{2} \cdot$ Giovanny Montoya ${ }^{3} \cdot$ Daniel Bin $\mathrm{Ng}^{4}$
}

Published online: 5 June 2019

(c) The Author(s) 2019

\begin{abstract}
Objectives The aim of this study was to evaluate the cost effectiveness of mirabegron relative to two antimuscarinics, oxybutynin extended release (ER) and tolterodine ER, in patients with overactive bladder (OAB) from the perspective of a third-party payer in Colombia.

Methods A Markov model simulated the therapeutic management, disease course, and complications in hypothetical cohorts of OAB patients over a 5-year period. The model predicted costs and three outcomes: quality-adjusted life-years (QALYs), micturition state improvement (MSI), and incontinence state improvement (ISI). In each 1-month cycle, patients could transition between different health states reflecting symptom severity. Transition probabilities were estimated from a published mirabegron trial and mixed treatment comparison. Other inputs such as treatment discontinuation based on treatment-specific rates of persistence, resource use and costs, anticholinergic burden, comorbidity treatment, and drug acquisition were obtained from Società Italiana Scienze Mediche, Instituto de Seguros Sociales Tariff Manual, published literature, and expert opinion. Deterministic and probabilistic sensitivity analyses were conducted. Costs are presented in 2017 Colombia Pesos (COP).

Results Mirabegron was cost effective for all outcome measures at a willingness-to-pay threshold of 124,919,725 COP, which is three times the per capita gross domestic product (GDP). Using QALYs as the measure of effect, mirabegron had an incremental cost-effectiveness ratio (ICER) of 85,802,036 COP/QALY (26,365 USD/QALY) and 66,360,134 COP/ QALY (20,384 USD/QALY) versus oxybutynin and tolterodine, respectively. Probabilistic sensitivity analyses showed that mirabegron was cost effective in $99.5 \%$ and $100 \%$ of simulations compared with oxybutynin and tolterodine, respectively. Using MSI and ISI as the measure of effects yielded ICERs below one GDP.

Conclusions Mirabegron is a cost effective alternative to oxybutynin and tolterodine from the perspective of a third-party payer in Colombia.
\end{abstract}

\section{Key Points for Decision Makers}

The results of our analysis demonstrate that mirabegron is cost effective compared with tolterodine extended release (ER) and oxybutynin ER from the perspective of a Colombian third-party payer.

Mirabegron confers more quality-adjusted life-years (QALYs) gained and better improvement in terms of micturition and incontinence, but is costlier than its comparators.

The higher drug acquisition cost of mirabegron is offset by greater improvement in quality of life and overactive bladder $(\mathrm{OAB})$ symptoms and fewer OAB-related comorbidity and adverse events. 


\section{Introduction}

Overactive bladder (OAB) is a condition characterized by urinary urgency often accompanied by frequency, nocturia, and sometimes urinary incontinence [1]. The prevalence of $\mathrm{OAB}$ in the United States (US) has been estimated in a 2005 survey of 20,000 men and women age 40 years and older at $15.8 \%$ and $32.6 \%$ in men and women, respectively, based on OAB symptoms categorized as occurring at least often [2]. In Venezuela, Brazil, and Argentina, the prevalence of OAB has been estimated at $21 \%$ in men and women between the age of 18 and 75 years [3], 24.4\% in men and women between the age of 16 and 55 years [4], and $23.4 \%$ in men and women [5], respectively.

Despite OAB being a prevalent condition, may patients receive no medical treatment. The estimated real-world OAB population that was seeking care was only about $60 \%$ in the general population in France, Germany, Italy, Spain, Sweden, and the United Kingdom [6]. Furthermore, 17.1\% and $28.6 \%$ of men and women who sought treatment aged 45 years and older in US-based IMS Health data received pharmacologic treatment [7].

OAB can have a profound impact on quality of life. It has been estimated that up to $50 \%$ of people with OAB experience depression and anxiety [8-11]. OAB can also negatively impact the ability to participate in physical activity, sleep, sexual activity, and work or employment and is associated with fatigue, erectile dysfunction, and falls [8, 12-15]. The breadth of adverse impacts associated with $\mathrm{OAB}$ contributes to the considerable economic impact of the condition [16-18].

Current first-line pharmacotherapy for OAB in Colombia includes antimuscarinics, a type of anticholinergic. Antimuscarinics have been associated with adverse effects (AEs) including dry mouth and constipation, which adversely affect treatment adherence and persistence and can have significant impacts for patients [19, 20]. Additionally, antimuscarinics contribute to the anticholinergic burden (ACB), which has been associated with mild cognitive impairment (MCI) and increased healthcare utilization [21-23]. Conversely, mirabegron is a first-in-class selective oral $\beta_{3}$-adrenoceptor agonist, and therefore does not contribute to ACB. Mirabegron has demonstrated similar efficacy as antimuscarinics but with improved tolerability in men and women $\geq 18$ years of age with symptoms of OAB for $\geq 3$ months [24-26] and, consequently, better persistence [27].

We developed a Markov model to analyze the cost effectiveness of mirabegron compared with oral antimuscarinic agents commonly prescribed for the treatment of adults with $\mathrm{OAB}$ in Colombia from a third-party payer perspective.

\section{Methods}

\subsection{Model Overview and Outcomes}

A Markov model was developed to analyze the cost effectiveness of mirabegron $50 \mathrm{mg}$ once daily (QD) versus two antimuscarinics commonly used in Colombia for the treatment of $\mathrm{OAB}$ in first-line: oxybutynin extended release (ER) $12 \mathrm{mg}$ on average QD and tolterodine ER $4 \mathrm{mg}$ QD. These comparators were selected based on expert opinion and clinical practice in Colombia [28]. Seven interviews were conducted among urologists to understand resource consumption related to $\mathrm{OAB}$. The clinical experts were chosen to have representation from the main city Bogota and a smaller city in the country (Cali). After the first round of interviews, the list of comparators was revised and a second round of interviews with the same physicians were conducted to solve inconsistencies. The model simulated the therapeutic management, course of disease, and complications in hypothetical cohorts of patients with $\mathrm{OAB}$, and its impact on costs and health outcomes. More specifically, the primary outcome was quality-adjusted life-years (QALYs) whereas improvement in frequency of micturition and incontinence were selected as secondary outcomes because they were the coprimary outcome measures of the pivotal mirabegron trials. The cycle length of the model was 1 month, and the time horizon was 5 years; real-world data showed that $75-90 \%$ of patients treated with mirabegron or antimuscarinics discontinue therapy within 1 year [27]. The model was programmed in Microsoft Excel 2016.

\subsection{Model Structure}

At model entry, patients were assigned to treatment with either mirabegron, tolterodine ER, or oxybutynin ER (Fig. 1) and were distributed across five levels of severity of micturition and incontinence frequency (Supplemental Table 1, see electronic supplementary material [ESM]). Each month, patients either improved (i.e., transitioned to a lower-severity category), stayed at the same level of severity, or worsened. Depending on the severity level, patients experienced OAB-related comorbidities and required incontinence pads. In addition, patients experienced AEs and the consequences of higher ACB depending on the treatment selected. The model accounted for treatment discontinuation/switch based on treatment-specific rates of persistence found in the literature. Upon discontinuation of first-line therapy, patients could switch to another oral OAB treatment, initiate onabotulinumtoxinA, or forego treatment (i.e., become 'untreated') (Fig. 1). Those who became untreated remained as such for the rest of the time horizon and experienced higher rates of comorbidities associated with $\mathrm{OAB}$ 
$1^{\text {st }}$ Line Treatment $2^{\text {nd }}$ Line Treatment $3^{\text {rd }}$ Line Treatment

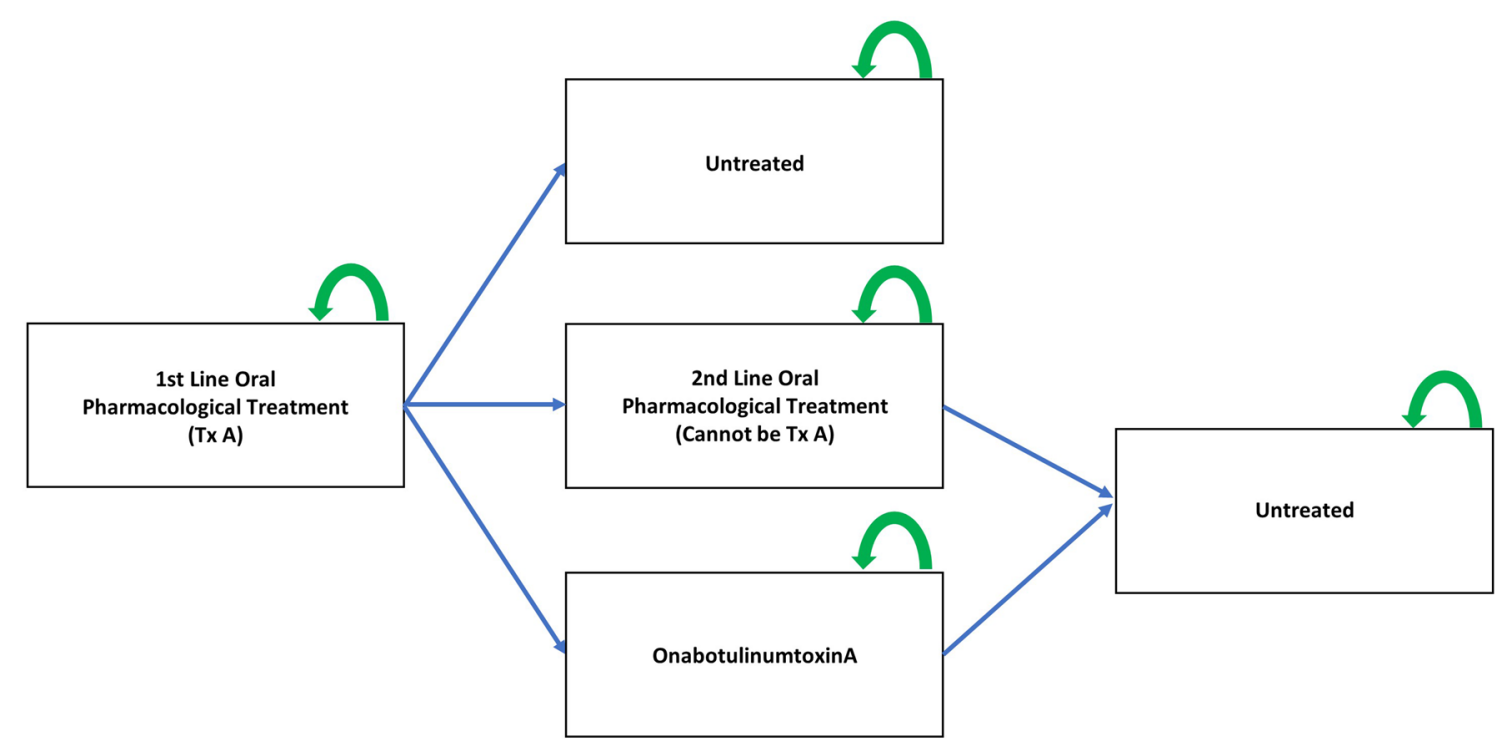

Fig. 1 Model flow diagram. $T x$ treatment

and increased incontinence pad usage but did not suffer from AEs or higher ACB. Discontinuation of second-line therapy led to permanent discontinuation. Those who switched to another oral $\mathrm{OAB}$ drug chose a treatment from a basket of $\mathrm{OAB}$ drugs, weighted by market share, which excluded the initial treatment. All consequences of second-line oral treatment were similarly weighted, including persistence, transition probabilities, $\mathrm{OAB}$ comorbidities, incontinence pad usage, AEs, and ACB consequences. Finally, those who switched to onabotulinumtoxinA either experienced a reduction of their symptoms (success) or returned to their initial symptom state (failure).

Costs were allocated throughout the model based on cost of $\mathrm{OAB}$ treatment, physician visits, monitoring, $\mathrm{ACB}, \mathrm{OAB}$ comorbidities, and incontinence pad usage, whereas each severity level of $\mathrm{OAB}$ symptoms was assigned a different quality-of-life value and utility decrements for AEs, comorbidities, and MCI were applied. Total costs and improvements in QALYs and OAB symptoms were calculated over the time horizon of the model from which cost per QALY, cost per micturition state improvement (MSI), and cost per incontinence state improvement (ISI) were derived.

\subsection{Model Input Parameters}

\subsubsection{Baseline Patient Distributions}

Initial age and gender distributions, presented in Supplemental Table 3 (see ESM), were based on expert opinion from clinicians in Colombia. Initial symptom severity distributions were based on SCORPIO trial data [29] (Supplemental Table 1 [see ESM]).

\subsubsection{Transition Probabilities}

Transition probabilities between severity levels for each symptom were obtained from a multinomial logistic regression model estimated from the SCORPIO clinical trial [24] and a mixed treatment comparison (MTC) of randomized controlled trials of all antimuscarinics (fesoterodine, solifenacin, tolterodine, oxybutynin, and trospium) [25], described previously [26, 29]. The SCORPIO trial data has shown that over the course of the 12-week trial, the improvement was greatest during the first 4 weeks of treatment, then progressively decreased during the next 8 weeks. This can be seen in Figs. 3 to 8 in the Mirabegron US package. Our estimated transition probabilities were estimated separately for each of the first 3 months of treatment. After three months, symptom levels were assumed to be constant (Supplemental Table 4 and Supplemental Table 5, see ESM).

\subsubsection{Treatment Discontinuation and Switch}

Treatment-specific discontinuation rates were based on real-world persistence levels found in the literature [27]. Briefly, a large portion of the cohort discontinued in the first month, followed by discontinuation at a constant hazard until a steady state was reached, representing the portion of 
the cohort for whom the medication had acceptable efficacy and tolerable AEs (Supplemental Table 2, see ESM). After discontinuation of first-line therapy, second-line therapy use was based on the survey of clinicians and conditional on the first-line treatment (Supplemental Table 2).

\subsubsection{OnabotulinumtoxinA Treatment}

Based on expert opinion from seven clinicians from Colombia, $40 \%$ of the cohort that switched to another treatment after discontinuing first-line therapy initiated onabotulinumtoxinA. The probability of symptom improvement was estimated at $57.5 \%$ [30]. Improvement translated into a reduction of one micturition state and three incontinence states based on the same study that reported a mean change in micturitions per day and urgency urinary incontinence episodes per day of -2.15 and -2.93 , respectively [30]. As the effect of onabotulinumtoxinA wears off, re-treatment is necessary. Re-treatment was assumed to occur every 6 months among responders based on the reported median duration of response of 19-24 weeks [31]. We further assumed that patients who benefit from onabotulinumtoxinA have the same benefit upon re-injections [32]. However, due to tolerability issues, only $75 \%$ of successful patients undergo repeat injections [32]. Finally, for patients whose symptoms do not improve, a 3-month waiting period is applied based on the minimum time to qualify for re-treatment [31], after which a second round of treatment is attempted [33]. Second failure led to permanent discontinuation (patient became 'untreated'). These assumptions are summarized in Supplemental Table 2 (see ESM).

\subsubsection{Adverse Events}

Two types of AEs were included: dry mouth and constipation. These were selected based on expert opinion that these events are most bothersome to patients and likely to drive treatment discontinuation [34]. Monthly probabilities of having an AE were derived from SCORPIO (mirabegron) or the MTC (tolterodine ER and oxybutynin ER) as reported by Nazir et al. [26] (Supplemental Table 2, see ESM). It was assumed that after stopping treatment patients had zero probability of having an AE. Finally, onabotulinumtoxinA AEs were ignored.

\subsubsection{Overactive Bladder (OAB)-Related Comorbidities}

Studies have shown that $\mathrm{OAB}$ is frequently associated with comorbidities such as depression, skin rash, and urinary tract infections (UTIs) [35, 36]; thus, these comorbidities were included in the model. Six-month probabilities of skin rash and UTI were taken from Arlandis-Guzman et al. [35] and converted into monthly probabilities. Monthly probabilities of depression were derived from 12-month probabilities from Irwin et al. [36]. The monthly probabilities of OABrelated comorbidities depended on the incontinence severity level and are shown in Supplemental Table 6 (see ESM).

\subsubsection{Anticholinergic Burden}

Both model comparators are antimuscarinics, a type of anticholinergic. An analysis of the Regenstrief Medical Record System (RMRS) and Indianapolis Dementia Screening and Diagnosis dataset carried out by the Regenstrief Institute demonstrated a positive association between utilization and increased ACB. Specifically, a one-increment increase in ACB resulted in 1.135 additional outpatient visits and 0.114 additional emergency room (ED) visits annually in the following year, adjusting for age, gender, race, and several comorbidities $[21,37]$. The same analysis provided inputs for probabilities of mild cognitive impairment (MCI) associated with elevated ACB. Both comparators are assumed to increase ACB score by 3, whereas mirabegron does not contribute to ACB [21, 37].

\subsubsection{Utilities}

Utility values according to symptom severity were derived from the Overactive Bladder Questionnaire based on pooled data from three 12 -week, randomized, doubleblind, parallel group, placebo-controlled, multi-center studies of the European, Australian, South African, Canadian, and US populations [24, 38, 39], and the algorithm described by Yang et al. [40] in the base case. Details on the methodology are presented in Desroziers et al. [41]. Briefly, they were based on micturition and incontinence frequencies, as well as age, gender, previous OAB treatment status, and body mass index. The utilities derived from the EuroQol five-dimensional questionnaire (EQ5D-3L) were explored as a scenario analysis. Disutilities associated with AEs, comorbidities, and MCI were also included (Supplemental Table 2, see ESM). Disutilities associated with AEs were applied for the duration of a cycle based on SCORPIO trial data as reported by Nazir et al. [26]. Disutilities associated with OAB-related comorbidities and MCI were taken from the literature [42-45]. 


\subsubsection{Resource Utilization and Costs}

Inputs for resources and costs are presented in Supplemental Table 2 (see ESM). Costs were evaluated from a thirdparty payer perspective and are presented in 2017 Colombian Pesos (COP) [46]. The cost-effectiveness analysis has been submitted to the regulatory agency in Colombia and costs were adjusted according to the required methodology: costs obtained from Instituto de Seguros Sociales (ISS) were adjusted $(+30 \%)$ per the Manual of Economic Evaluation in Colombia [47]. Resource utilization and costs were categorized into the following categories:

1. Drug-related We assumed that patients prescribed an oral OAB took one pill each day every month until discontinuation of the treatment. Drug wastage or partial compliance were not considered. The model assumed one physician visit at treatment initiation and upon switching to second-line oral treatment. For onabotulinumtoxinA, drug-related costs included the acquisition cost [48], injection cost (PB 861411) [49], as well as one physician visit per injection. Finally, each treatment option was associated with a monthly monitoring cost calculated as the product of resource utilization obtained via expert opinion and corresponding unit cost [49].

2. OAB-related comorbidities Direct costs of OAB-related comorbidities were taken from the literature [50-52].

3. Anticholinergic burden Each increase in ACB has been associated with 1.135 and 0.114 additional outpatient and ED visits annually, respectively, per increment in ACB [21, 37]. Costs of ED visits (PB I39134) and outpatient visits (PB 890301) were taken from ISS [49].

4. Incontinence pad usage Based on SCORPIO trial data, the daily incontinence pad use was assumed to increase with the incontinence severity level, and to be independent of OAB treatment [29]. Cost per pad was taken from a published source [53].

\subsection{Discount Rate}

A rate of 5\% was used to discount costs and utilities in the base-case analysis. The two secondary measures of effects (i.e., improvement in micturition and incontinence severity state levels) were not discounted by construction. More specifically, improvement was calculated based on the difference between the average micturition or incontinence score at baseline and at the end of the time horizon. The symptom scores were calculated using the distribution of patients in each of the five severity levels and assigning a score of 0-4 to each severity level. Because the change in score is not based on the cumulative frequency of symptoms over the time horizon, it was deemed inappropriate to discount the secondary measure of effects.

\subsection{Model Outputs}

The primary cost-effectiveness measure was the incremental cost-effective ratio (ICER), expressed as cost per QALY gained. We used a willingness-to-pay threshold (WTP) of $124,919,725$ COP/QALY gained, which is three times the per capita gross domestic product (GDP) of Colombia [54], as recommended by the Manual of Economic Evaluation in Colombia [47]. Secondary cost effectiveness measures were COP/MSI gained and COP/ISI gained using the same WTP threshold. Other outputs reported include costs of each treatment option stratified by OAB drug costs, medical costs, and incontinence pad costs; average improvement in OAB symptoms (ISI and MSI) and clinical outcomes such as AE and comorbidity counts; and treatment status at the end of the time horizon.

\subsection{Sensitivity Analyses}

To assess the impact of uncertainty on our base-case estimates, sensitivity analyses were performed including deterministic sensitivity analyses (DSA), probabilistic sensitivity analyses (PSA), and specific scenario analyses. Supplemental Table 2 shows the range used in the DSA as well as the types of distribution and associated parameters used for the PSA (see ESM).

\subsection{Model Validation}

The model underwent verification and validation consistent with recommendations by Philips et al. [55] and the ISPOR Task Force [56].

\section{Results}

\subsection{Base-Case Results}

Table 1 presents the total costs (OAB drug treatment, medical costs, and incontinence pad costs), and outcomes (i.e., QALYs, ISI, and MSI) for each treatment option using the base-case parameter input values. The corresponding cost-effectiveness planes are presented in Supplemental Fig. 1 through Supplemental Fig. 3 (see ESM). For all three measures of effectiveness, oxybutynin ER was the least expensive treatment, followed by tolterodine ER, and mirabegron. In terms of efficacy, oxybutynin ER offered the least improvement in effects, whereas mirabegron delivered the greatest improvement.

Using our primary outcome (i.e., QALYs), mirabegron had an ICER of 85.8 million COP/QALY and 66.4 million COP/QALY versus oxybutynin ER and tolterodine $\mathrm{ER}$, respectively. In terms of incontinence outcomes, mirabegron had an ICER of 33.8 million COP/ISI and 
Table 1 Cost-effectiveness results in the base-case analysis

\begin{tabular}{|c|c|c|c|c|c|}
\hline Outcome & Mirabegron & Oxybutynin ER & $\begin{array}{l}\text { Incremental (Mir vs } \\
\text { Oxy) }\end{array}$ & Tolterodine ER & $\begin{array}{l}\text { Incremental } \\
\text { (Mir vs Tol) }\end{array}$ \\
\hline \multicolumn{6}{|l|}{ Total costs (COP) } \\
\hline $\mathrm{OAB}$ drug costs & $2,478,026$ & 918,698 & $1,559,328$ & $1,375,128$ & $1,102,898$ \\
\hline Medical costs & $5,085,145$ & $5,172,529$ & $-87,384$ & $5,178,772$ & $-93,628$ \\
\hline Incontinence pad costs & $2,206,254$ & $2,306,463$ & $-100,210$ & $2,290,709$ & $-84,455$ \\
\hline Total & $9,769,424$ & $8,397,690$ & $1,371,735$ & $8,844,609$ & 924,815 \\
\hline \multicolumn{6}{|l|}{ Total costs (USD) ${ }^{\mathrm{a}}$} \\
\hline $\mathrm{OAB}$ drug costs & 761 & 282 & 479 & 422 & 339 \\
\hline Medical costs & 1562 & 1589 & -27 & 1591 & -29 \\
\hline Incontinence pad costs & 678 & 708 & -31 & 704 & -26 \\
\hline Total & 3001 & 2580 & 421 & 2717 & 284 \\
\hline \multicolumn{6}{|l|}{ Total effects } \\
\hline QALYs & 3.455 & 3.439 & 0.016 & 3.441 & 0.014 \\
\hline ISI & 0.200 & 0.160 & 0.041 & 0.166 & 0.034 \\
\hline MSI & 0.201 & 0.153 & 0.048 & 0.160 & 0.041 \\
\hline \multicolumn{6}{|l|}{ ICER (COP million) } \\
\hline ICER, cost per QALY gained & & & 85.8 & & 66.4 \\
\hline ICER, cost per ISI & & & 33.8 & & 26.9 \\
\hline ICER, cost per MSI & & & 28.7 & & 22.4 \\
\hline \multicolumn{6}{|l|}{$\operatorname{ICER}(\mathrm{USD})^{\mathrm{a}}$} \\
\hline ICER, cost per QALY gained & & & 26,356 & & 20,384 \\
\hline ICER, cost per ISI & & & 10,397 & & 8275 \\
\hline ICER, cost per MSI & & & 8806 & & 6876 \\
\hline
\end{tabular}

COP Colombian Pesos, ICER incremental cost-effectiveness ratio, ISI incontinence state improvement, Mir mirabegron, MSI micturition state improvement, $O A B$ overactive bladder, $O x y$ oxybutynin extended release, $Q A L Y s$ quality-adjusted life-years, Tol tolterodine extended release, USD US dollars

${ }^{a}$ The exchange rate from COP to USD used was 0.000307199 USD/COP. Source: https://www.xe.com/currencyconverter/convert/?Amoun $\mathrm{t}=1 \&$ From $=$ USD\&To=COP (Last accessed: May 6, 2019 4:30PM ET)

26.9 million COP/ISI versus oxybutynin ER and tolterodine ER, respectively. Using MSI as the effect measure, mirabegron had an ICER of 28.7 million COP/MSI and 22.4 million COP/MSI versus oxybutynin ER and tolterodine ER, respectively. Assuming a WTP threshold of three times the GDP of Colombia (124.9 million COP), mirabegron was cost effective compared with both comparators, regardless of the OAB outcome measure applied. Furthermore, using the two secondary outcome measures, the comparison of mirabegron against oxybutynin ER and tolterodine ER yielded ICERs below one times the per capita GDP.

The model also estimated relevant clinical outcomes (Supplemental Table 7, see ESM). A slightly higher proportion of patients remained on their first-line therapy at the end of the time horizon when initiating with mirabegron (10.5\% vs $6.9 \%$ for oxybutynin ER and $7.7 \%$ for tolterodine ER) and fewer discontinued treatment. Also, mirabegron resulted in the fewest comorbidity-related events across all three comorbidities included in the model. In terms of treatment-related AEs, initiating treatment with mirabegron reduced the frequency of dry mouth but not constipation.

\subsection{Deterministic Sensitivity Analyses}

The results of the DSA depicted as tornado diagrams showing the 20 most influential model parameters on the ICER comparing mirabegron with oxybutynin ER and tolterodine ER are shown in Fig. 2 and Supplemental Fig. 4, respectively. The results are shown with QALYs as the outcome measure, though results were similar for MSI and ISI (data not shown). For both comparisons, the model was most sensitive to the persistence rate of the initial treatment, the utility decrement associated with MCI and dry mouth, onabotulinumtoxinA-related parameters, the rate of dry mouth, and the monitoring cost associated with each treatment option. In all analyses, mirabegron remained cost effective at the 124.9 million COP per QALY threshold. 


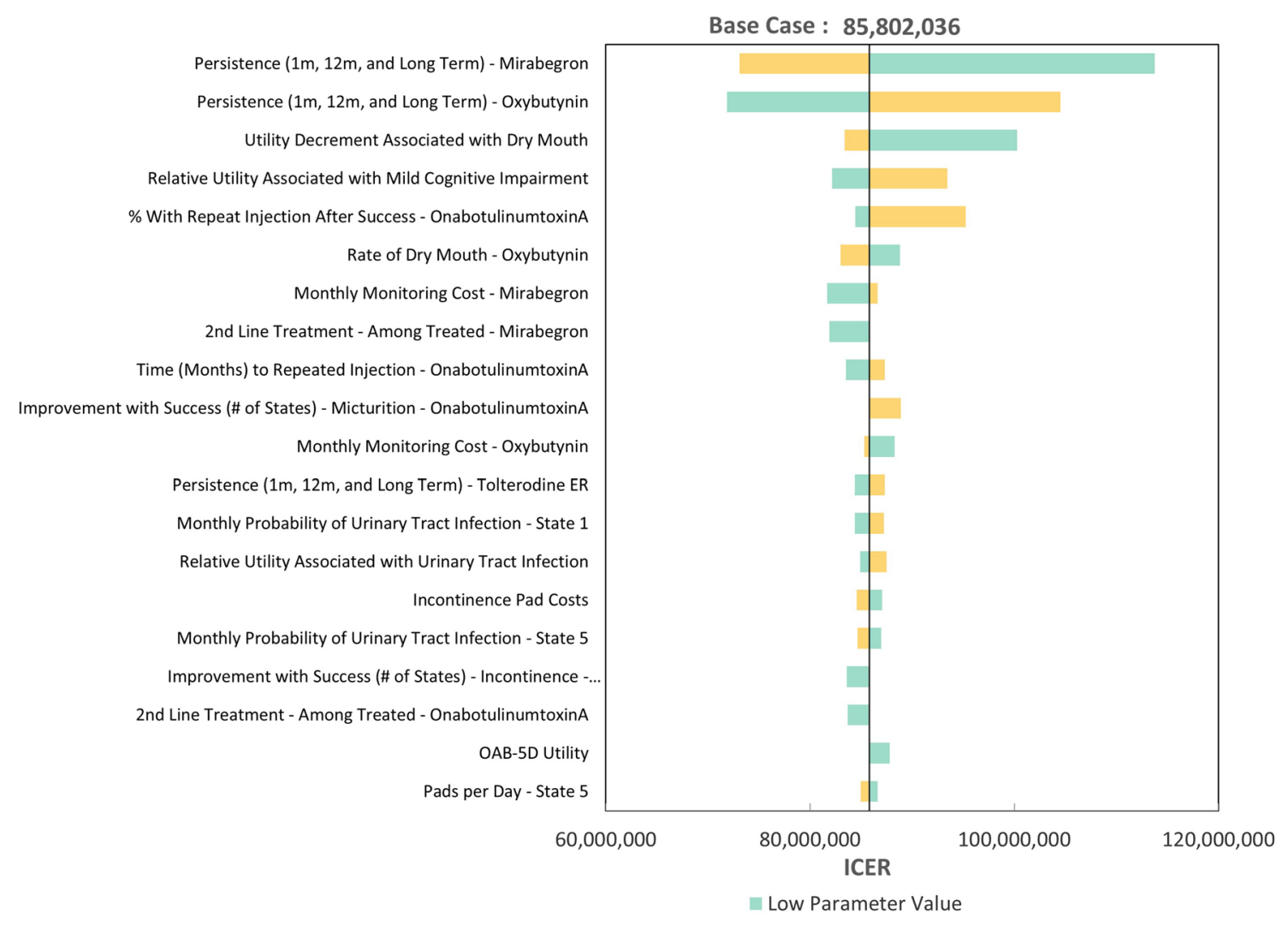

Fig. 2 DSA results showing the 20 most influential model parameters comparing mirabegron and oxybutynin ER: QALYs. DSA deterministic sensitivity analysis, ER extended release, ICER incremental cost-effectiveness ratio, QALYs quality-adjusted life-years

\subsection{Probabilistic Sensitivity Analyses}

The PSA estimated that the probability of mirabegron being cost effective against oxybutynin ER and tolterodine ER was $99.5 \%$, and $100 \%$, respectively, at the three times the per capita GDP WTP threshold. This is depicted two ways: a scatterplot of all 1000 simulations with the line corresponding to the WTP threshold (Fig. 3 and Supplemental Fig. 5) and the corresponding cost-effectiveness acceptability curve (CEAC) (Fig. 4 and Supplemental Fig. 6). As shown in the PSA scatterplots, all simulations appear below the 124.9 million WTP threshold line when comparing against tolterodine ER and most appear below the WTP threshold line when comparing against oxybutynin ER.

\subsection{Scenario Analyses}

To further assess the robustness of the cost-effectiveness results, several scenario analyses were conducted. Table 2 presents each scenario with the resulting incremental costs, QALYs, and corresponding ICERs for the comparison of mirabegron versus oxybutynin ER. Tolterodine ER was extendedly dominated in all scenarios and thus was excluded from the table. For all scenarios, mirabegron remained cost effective at the three times per capita GDP WTP threshold with ICERs ranging from 84.4 million (no discounting) to 121.4 million (EQ-5D utilities). The corresponding results using our secondary outcomes (i.e., ISI and MSI) further strengthened our findings that mirabegron is a cost-effective option, regardless of the scenario analysis conducted, with most ICERs below one times the per capita GDP WTP and all below three times the per capita GDP WTP threshold (data not shown).

\section{Discussion}

The results of our analysis suggest that mirabegron is cost effective compared with tolterodine ER and oxybutynin ER from the perspective of a third-party payer in Colombia. The higher drug acquisition cost of mirabegron compared with its comparators was offset by greater improvement in quality of life and OAB symptoms, fewer OAB-related comorbidity events, reduced frequency of dry mouth and incontinence pad usage, improved persistence, and reduced ACB.

Our model calculated an ICER of 85.8 million COP/ QALY and 66.4 million COP/QALY for mirabegron vs oxybutynin ER and tolterodine ER, respectively, both of 


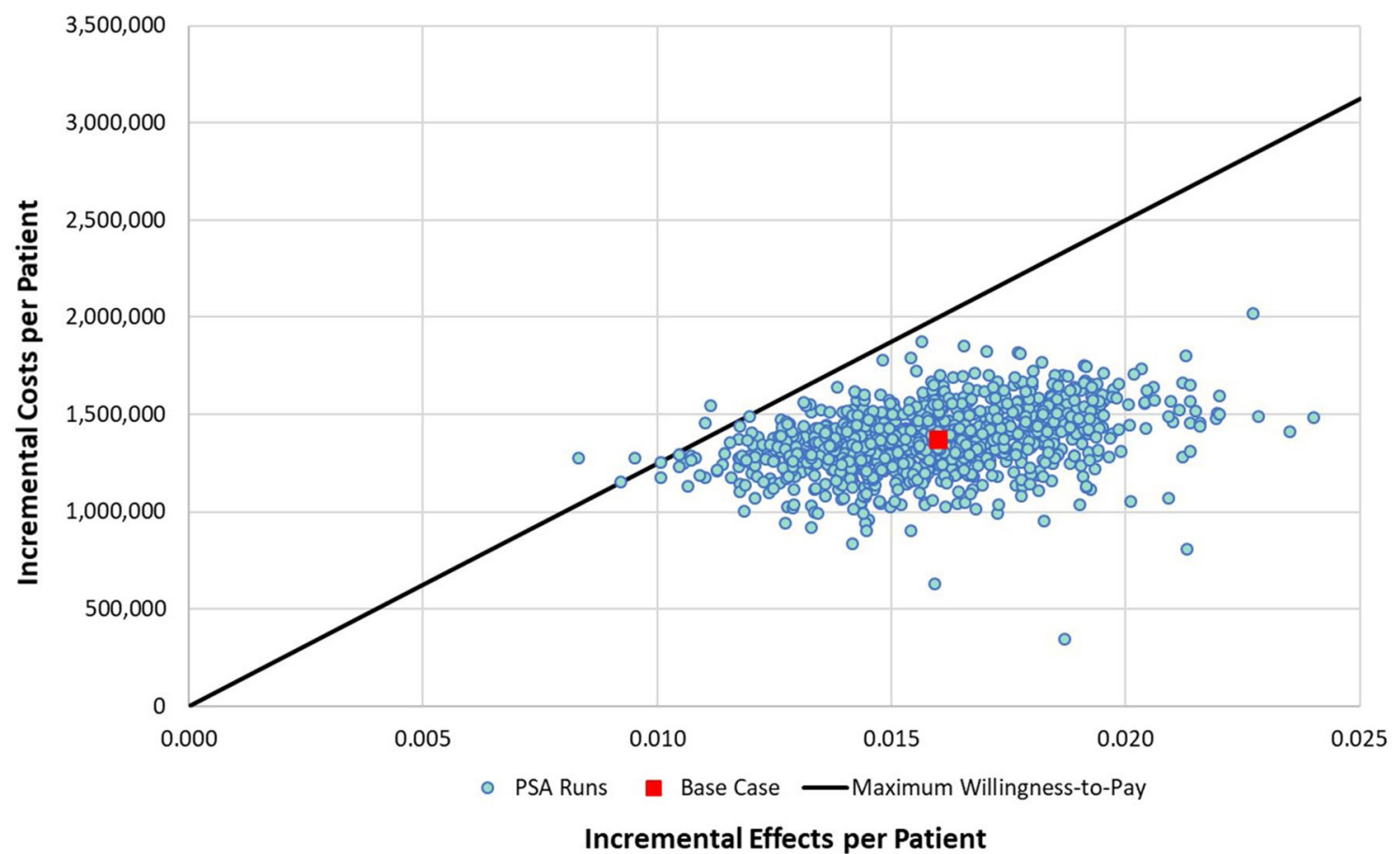

Fig. 3 PSA results, scatterplot of incremental cost-effectiveness, mirabegron vs oxybutynin ER: QALYs. ER extended release, PSA probabilistic sensitivity analysis, $Q A L Y s$ quality-adjusted life-years

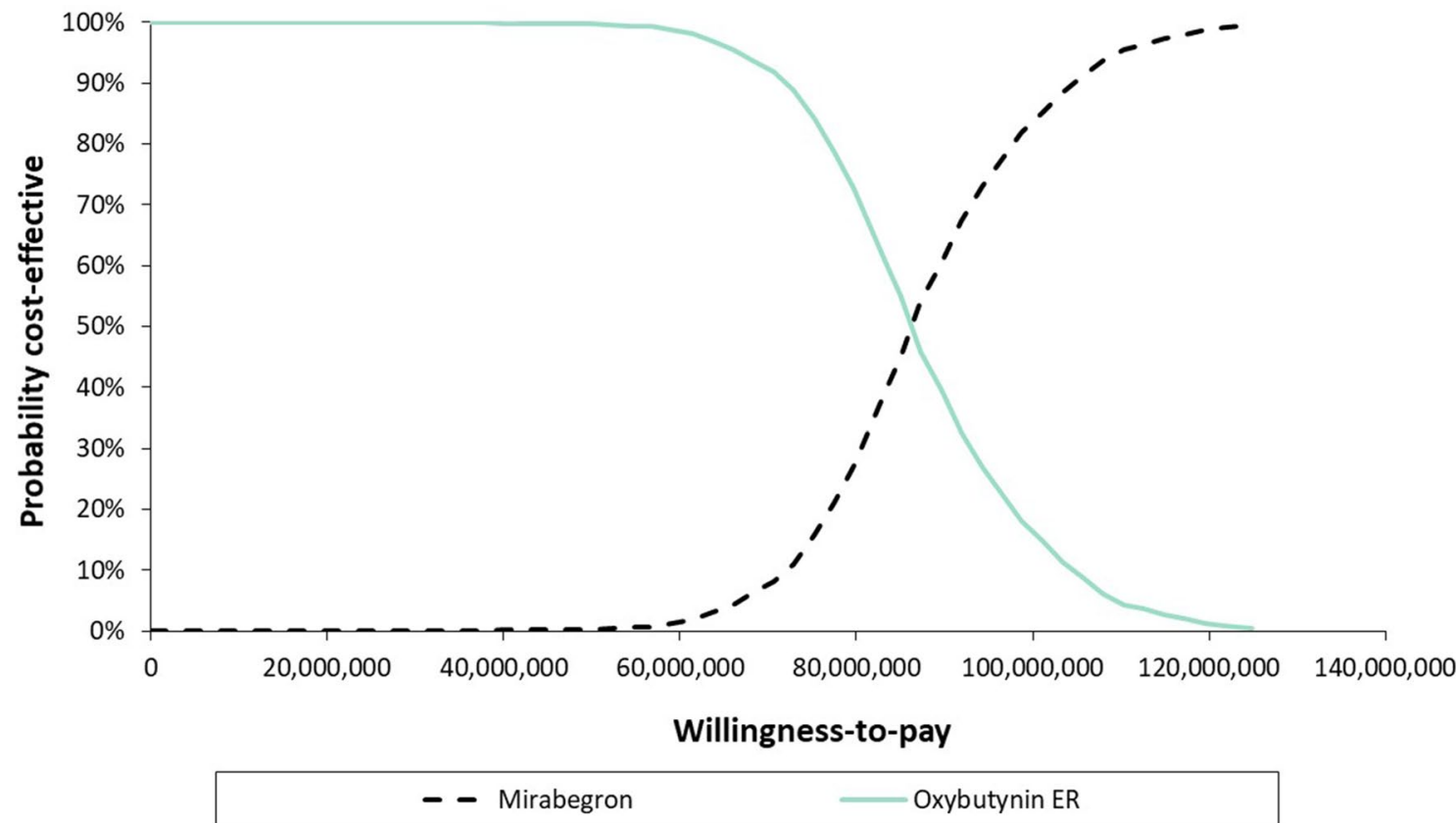

Fig. 4 PSA results, CEAC, mirabegron vs oxybutynin ER: QALYs. CEAC cost-effectiveness acceptability curve, ER extended release, $P S A$ probabilistic sensitivity analysis, $Q A L Y s$ quality-adjusted life-years

which are below the 124.9 million COP WTP threshold. ICERs remained well below the WTP threshold using ISI and MSI as measures of effects. Comprehensive sensitivity analyses, in the form of PSA, DSA, and scenario analyses were conducted to explore the impact of parameters and any structural uncertainty within the model. These concluded that our base-case results are robust and the results also align with two previously published cost-effectiveness analyses 
Table 2 Cost-effectiveness results in the base case vs scenario analyses, mirabegron vs oxybutynin ER: QALYs

\begin{tabular}{llll}
\hline Scenario & $\begin{array}{l}\text { Incremental cost } \\
(\mathrm{COP})\end{array}$ & $\begin{array}{l}\text { Incremental } \\
\text { QALY }\end{array}$ & $\begin{array}{l}\text { ICER (COP/ } \\
\text { QALY } \\
\text { gained) }\end{array}$ \\
\hline Base case & $1,371,735$ & 0.016 & 85.8 \\
Time horizon of 3 years & $1,105,660$ & 0.012 & 90.3 \\
Time horizon of 10 years & $1,879,759$ & 0.022 & 85.5 \\
Discount rate for costs and utilities of 0\% & $1,476,749$ & 0.017 & 84.4 \\
Discount rate for costs and utilities of 3.5\% & $1,401,159$ & 0.016 & 85.4 \\
Discount rate for costs and utilities of 7\% & $1,334,957$ & 0.015 & 86.3 \\
Discount rate for costs and utilities of 12\% & $1,253,687$ & 0.014 & 87.7 \\
$100 \%$ male & $1,347,045$ & 0.016 & $85,967,000$ \\
100\% female & $1,397,869$ & 0.016 & 87.2 \\
EQ-5D utilities & $1,371,735$ & 0.011 & 121.4 \\
Same efficacy & $1,421,687$ & 0.013 & 113.0 \\
\hline
\end{tabular}

$C O P$ Colombian Pesos, EQ-5D EuroQol five-dimensional questionnaire, ER extended release, ICER incremental cost-effectiveness ratio, $Q A L Y s$ quality-adjusted life-years of mirabegron [26, 37]. Nazir et al. compared, from the perspective of the United Kingdom (UK) National Health Service (NHS), the cost effectiveness of mirabegron with numerous antimuscarinic therapies commonly used to treat $\mathrm{OAB}$ in the UK [26]. They found that mirabegron was cost effective compared with antimuscarinics due to improved persistence and quality of life resulting from lower incidence of AEs [26]. Another analysis evaluating mirabegron against six different antimuscarinic therapies from a US-based commercial health plan and Medicare Advantage perspective similarly found that mirabegron was cost effective compared with several antimuscarinic therapies, which was primarily driven by lower healthcare resource use due to lower ACB [37]. Similar to our analysis, a Markov model was used to track transitions between symptom severity levels, based upon a model described by Kobelt et al. [57]. Taken together, these results support the assertion that mirabegron is a cost effective alternative to antimuscarinics for the treatment of OAB from a variety of payer perspectives.

Our study has many strengths. Our model structure is based on previously published cost-effectiveness models that have been reviewed and deemed acceptable [58]. A review of published economic models evaluating OAB pharmacotherapies found that no standard exists for defining $\mathrm{OAB}$ health states, although most include a measure of incontinence frequency like the current study [59]. Other strengths of our model include the quality of the SCORPIO trial data, the inclusion of utilities based on both OAB-5D and EQ-5D instruments, the inclusion of ACB as a factor in the analysis (as mirabegron does not share the ACB associated with antimuscarinic pharmacological OAB treatments), and finally the inclusion of the latest 2017 persistence data for oral OAB therapies [27].

Our study also has limitations. One limitation is that transition probabilities were estimated from SCORPIO for mirabegron and tolterodine ER. For oxybutynin ER, probabilities were obtained using a calibration method based on results of an MTC assessing the effectiveness of antimuscarinics. However, the MTC only provided estimates of mean changes in micturition and incontinence episodes for each treatment included in the analysis. Also, there is no unique solution to the calibration problem. To address this potential limitation, cost-effectiveness results were regenerated discarding the MTC and instead assuming both comparators have the same efficacy as mirabegron. In this scenario analysis, tolterodine ER remained extendedly dominated and the ICER of mirabegron against oxybutynin ER was higher than our base-case findings, but still below the accepted WTP threshold. Additionally, utility estimates are not based on the Colombian population but rather were derived from the three 12-week, randomized, double-blind, parallel group, placebo controlled, multi-center studies of mirabegron in the European, Australian, South African, Canadian, and US populations.

A simplifying assumption of the analysis concerns patients who forego oral OAB treatment after discontinuing first- and second-line treatment. We assumed these patients remain untreated for the rest of the model cycles and furthermore, patients could not resume their previous therapy. These assumptions might not accurately reflect the treatment patterns in Colombia. In addition, no data exist to inform the proportion of patients who would initiate mirabegron after discontinuation of an antimuscarinic. Sensitivity analyses demonstrated that varying our base-case assumptions does not significantly impact the results.

Several assumptions related to onabotulinumtoxinA are subject to uncertainty: whether or not clinicians would try a second dose prior to discontinuing therapy, the time between injections, the proportion of patients discontinuing onabotulinumtoxinA despite experiencing a success, and 
the probability of success/failure with onabotulinumtoxinA. Furthermore, success was modeled as an improvement in terms of a change in micturition and incontinence states as opposed to more precise time-dependent transition probabilities due to the unavailability of patient-level clinical trial data. Extensive deterministic and probabilistic sensitivity analyses were undertaken to assess the impact of varying the onabotulinumtoxinA treatment-related parameters. In all cases, mirabegron remained cost effective.

The three comorbidities included in the model, depression, skin rash and UTI, are closely associated with levels of incontinence. A limitation of the model is the source of probabilities for comorbidities, which reported probabilities for a binary incontinence outcome-controlled or uncontrolled. If left as a binary outcome, only complete relief from incontinence would decrease the probability of comorbidities. Thus, the probabilities were transformed from a binary (controlled/uncontrolled) to continuous distribution by assuming a linear relationship with incontinence episodes and assuming the highest severity level in our model was the uncontrolled state.

Our estimate of the impact of ACB may be limited as it is based on an analysis quantifying the relationship between health utilization and ACB that only included oxybutynin [37]. We nonetheless feel that it is appropriate to use these data in our analysis. Evidence of the influence of ACB on cognitive and physical outcomes has been consistent [21, 22, 60, 61]. Moreover, all OAB antimuscarinics have the same ACB designation as anticholinergics that may cause delirium or chronic cognitive impairment [21, 62]. The effect of elevated ACB and its associated risk of cognitive impairment is a concern for the elderly population [22, 63]. To be conservative, the model excludes the possible association of ACB with increased mortality, even though a positive association has been documented in a large prospective study [60].

The model considered only two AEs, dry mouth and constipation, on the basis of a report showing these events are most likely to cause treatment switch and are therefore the main drivers of AE-related discontinuation [34]. Considering the AEs reported most frequently with mirabegron occur at a similar incidence with placebo, we believe limiting the list of AEs to dry mouth and constipation was unlikely to bias the analysis [24]. Moreover, AEs related to onabotulinumtoxinA, such as urinary retention and UTIs, were excluded for simplicity. Including such AEs would likely reduce the ICERs because a larger proportion of patients initiating on an antimuscarinic switched to onabotulinumtoxinA due to poorer persistence compared with mirabegron.

Finally, the base-case model relied on OAB-5D utilities as opposed to the EQ-5D utilities. OAB-5D utilities were found to be similar to EQ-5D utilities for the most severe health states, but higher than EQ-5D utilities for less severe health states. Thus, the range of OAB-5D utilities is wider than the range of EQ-5D utilities, leading to more favorable ICERs. Published studies documented that the EQ-5D has a number of potential limitations, such as the lack of sensitivity in specific disease contexts [64-66]. Furthermore, the equations in Desroziers et al. explained a substantially higher proportion of variance with OAB-5D than with EQ-5D utilities $(26.1 \%$ vs $5.1 \%)$ [41]. Thus, OAB-5D-derived utilities were used in the base case. We tested this assumption in a scenario analysis. Using the EQ-5D utilities yielded ICERs that were higher than the base-case estimates. Nevertheless, mirabegron remained a cost-effective option.

\section{Conclusions}

The current cost-effectiveness analysis model includes ACB, treatment persistence, and costly incontinence pad usage as factors in the analysis. Based on the results of our cost-effectiveness analysis, mirabegron confers more QALY gained and better improvement in terms of micturition and incontinence, albeit at a higher cost than its comparators, oxybutynin ER and tolterodine ER. Taken together, mirabegron appears to be cost effective against both comparators at a WTP threshold of 124.9 million COP for all effects: QALYs, ISI and MSI. Our results are robust in deterministic and probabilistic sensitivity analyses, as well as scenario analyses, thus strengthening our findings that mirabegron is a cost-effective solution to the treatment of $\mathrm{OAB}$ in Colombia.

Author contributions HP was responsible for programming the Microsoft Excel model, drafting of the manuscript, and statistical analyses. $\mathrm{RE}$ was responsible for drafting of the manuscript and administrative, technical, and material support. KD was responsible for validating the Microsoft Excel model results and providing quality control. PA provided study concept and design and study supervision. GM MD, MSc was responsible for acquiring Colombia-specific data, analysis and interpretation of data, study concept, and design. DBN PharmD, MBA, MPH was responsible for drafting of the manuscript, study concept and design and study supervision.

\section{Compliance with ethical standards}

Funding This study was initiated and funded by Astellas Pharma Global Development, Inc. Hélène Parise and Katherine Dea are contractors for Medicus Economics LLC, which received payment from Astellas to assist with data analysis. Robert Espinosa is an employee of Medicus Economics LLC. Pablo Anaya is an employee of IQVIA. Giovanny Montoya is an employee of Astellas Farma Colombia S.A.S. Daniel Ng is an employee of Astellas Pharma Global Development Inc.

Data availability statement Anonymized individual participant-level data analyzed for the current study meets one or more of the exceptions described on http://www.clinicalstudydatarequest.com under "Sponsor Specific Details for Astellas" and therefore are not publicly available. The Microsoft Excel model generated during the current study is available from corresponding author on reasonable request. 
Open Access This article is distributed under the terms of the Creative Commons Attribution-NonCommercial 4.0 International License (http://creativecommons.org/licenses/by-nc/4.0/), which permits any noncommercial use, distribution, and reproduction in any medium, provided you give appropriate credit to the original author(s) and the source, provide a link to the Creative Commons license, and indicate if changes were made.

\section{References}

1. Haylen BT, de Ridder D, Freeman RM, et al. An International Urogynecological Association (IUGA)/International Continence Society (ICS) joint report on the terminology for female pelvic floor dysfunction. Neurourol Urodyn. 2010;29(1):4-20.

2. Coyne KS, Sexton CC, Vats V, Thompson C, Kopp ZS, Milsom I. National community prevalence of overactive bladder in the United States stratified by sex and age. Urology. 2011;77(5):1081-7.

3. Davila HA, Lopez V, Nieves L, et al. Demographic distribution and prevalence of overactive bladder in Venezuela. Actas Urol Esp. 2010;34(2):176-80.

4. Teloken C, Caraver F, Weber FA, et al. Overactive bladder: prevalence and implications in Brazil. Eur Urol. 2006;49(6):1087-92.

5. Garcia Salord J, Belén R, Bevilacqua O. Epidemiología de la vejiga hiperactiva sobre una población de 3.692 consultas urológicas y 2.030 estudios urodinámicos. Rev Argent Urol. 2005;70(1):8-13.

6. Milsom I, Abrams P, Cardozo L, Roberts RG, Thuroff J, Wein AJ. How widespread are the symptoms of an overactive bladder and how are they managed? A population-based prevalence study. BJU Int. 2001;87(9):760-6.

7. Helfand BT, Evans RM, McVary KT. A comparison of the frequencies of medical therapies for overactive bladder in men and women: analysis of more than 72 million aging patients. Eur Urol. 2010;57(4):586-91.

8. Coyne KS, Sexton CC, Irwin DE, Kopp ZS, Kelleher CJ, Milsom I. The impact of overactive bladder, incontinence and other lower urinary tract symptoms on quality of life, work productivity, sexuality and emotional well-being in men and women: results from the EPIC study. BJU Int. 2008;101(11):1388-95.

9. Coyne KS, Sexton CC, Kopp ZS, Ebel-Bitoun C, Milsom I, Chapple C. The impact of overactive bladder on mental health, work productivity and health-related quality of life in the UK and Sweden: results from EpiLUTS. BJU Int. 2011;108(9):1459-71.

10. Milsom I, Kaplan SA, Coyne KS, Sexton CC, Kopp ZS. Effect of bothersome overactive bladder symptoms on healthrelated quality of life, anxiety, depression, and treatment seeking in the United States: results from EpiLUTS. Urology. 2012;80(1):90-6.

11. Nicolson P, Kopp Z, Chapple CR, Kelleher C. It's just the worry about not being able to control it! A qualitative study of living with overactive bladder. Br J Health Psychol. 2008;13(Pt 2):343-59.

12. Coyne KS, Sexton CC, Clemens JQ, et al. The impact of OAB on physical activity in the United States: results from OAB-POLL. Urology. 2013;82(4):799-806.

13. Coyne KS, Sexton CC, Thompson C, Kopp ZS, Milsom I, Kaplan SA. The impact of OAB on sexual health in men and women: results from EpiLUTS. J Sex Med. 2011;8(6):1603-15.

14. Darkow T, Fontes CL, Williamson TE. Costs associated with the management of overactive bladder and related comorbidities. Pharmacotherapy. 2005;25(4):511-9.

15. Hawken N, Hakimi Z, Aballéa S, Nazir J, Odeyemi IAO, Toumi M. Elicitation of health-related quality-of-life concepts associated with overactive bladder: a qualitative study. JHEOR. 2016;4(2):127-40.

16. Irwin DE, Mungapen L, Milsom I, Kopp Z, Reeves P, Kelleher C. The economic impact of overactive bladder syndrome in six Western countries. BJU Int. 2009;103(2):202-9.

17. Sacco E, Tienforti D, D'Addessi A, et al. Social, economic, and health utility considerations in the treatment of overactive bladder. Open Access J Urol. 2010;2:11-24.

18. Yehoshua A, Chancellor M, Vasavada S, et al. Health resource utilization and cost for patients with incontinent overactive bladder treated with anticholinergics. J Manag Care Spec Pharm. 2016;22(4):406-13.

19. Abrams P, Andersson KE, Buccafusco JJ, et al. Muscarinic receptors: their distribution and function in body systems, and the implications for treating overactive bladder. Br J Pharmacol. 2006;148(5):565-78.

20. Oefelein MG. Safety and tolerability profiles of anticholinergic agents used for the treatment of overactive bladder. Drug Saf. 2011;34(9):733-54.

21. Campbell NL, Perkins AJ, Bradt P, et al. Association of anticholinergic burden with cognitive impairment and health care utilization among a diverse ambulatory older adult population. Pharmacotherapy. 2016;36(11):1123-31.

22. Gray SL, Anderson ML, Dublin S, et al. Cumulative use of strong anticholinergics and incident dementia: a prospective cohort study. JAMA Intern Med. 2015;175(3):401-7.

23. Richardson K, Fox C, Maidment I, et al. Anticholinergic drugs and risk of dementia: case-control study. BMJ (Clin Res ed). 2018;361:k1315.

24. Khullar V, Amarenco G, Angulo JC, et al. Efficacy and tolerability of mirabegron, a beta(3)-adrenoceptor agonist, in patients with overactive bladder: results from a randomised European-Australian phase 3 trial. Eur Urol. 2013;63(2):283-95.

25. Maman K, Aballea S, Nazir J, et al. Comparative efficacy and safety of medical treatments for the management of overactive bladder: a systematic literature review and mixed treatment comparison. Eur Urol. 2014;65(4):755-65.

26. Nazir J, Maman K, Neine ME, et al. Cost-effectiveness of mirabegron compared with antimuscarinic agents for the treatment of adults with overactive bladder in the United Kingdom. Value Health J Int Soc Pharmacoecon Outcomes Res. 2015;18(6):783-90.

27. Wagg AS, Foley S, Peters J, Nazir J, Kool-Houweling L, Scrine L. Persistence and adherence with mirabegron vs antimuscarinics in overactive bladder: Retrospective analysis of a UK General Practice prescription database. Int J Clin Pract. 2017;71(10):e12996. https://doi.org/10.1111/ijcp.12996.

28. Sociedad Colombiana De Urologia. Guias De Practica Clinica (GPC). http://scu.org.co/userfiles/file/guias2015/6-\%20GUIA\%20 VEJIGA\%20HIPERACTIVA\%20NO\%20NEUROGENICA.pdf. Accessed 11 Jan 2018.

29. NICE. Mirabegron for the treatment of overactive bladder. Single technology appraisal [STA]. London; 2012.

30. Nitti VW, Dmochowski R, Herschorn S, et al. OnabotulinumtoxinA for the treatment of patients with overactive bladder and urinary incontinence: results of a phase 3, randomized, placebo controlled trial. J Urol. 2013;189(6):2186-93.

31. Allergan. BOTOX (onabotulinomtoxinA) [package insert]. U.S. Food and Drug Administration website. https://www.acces sdata.fda.gov/drugsatfda_docs/label/2017/103000s53021bl.pdf. Revised April 2017. Accessed 10 Nov 2018.

32. Wu JM, Siddiqui NY, Amundsen CL, Myers ER, Havrilesky LJ, Visco AG. Cost-effectiveness of botulinum toxin a versus anticholinergic medications for idiopathic urge incontinence. J Urol. 2009;181(5):2181-6. 
33. Hepp Z, Yehoshua A, Gultyaev D, Lister J. Cost effectiveness of onabotulinumtoxina versus Ptns and Sns for the treatment of overactive bladder from the US payer perspective. Value Health. 2016;19(3):A130-1.

34. Compion G, Jackson J, Janes J. 691 Reasons for switching antimuscarinic therapy: results from a European cross-sectional survey of physicians and patients with OAB. Eur Urol Suppl. 2012;11(1):e691-e691a. https://doi.org/10.1016/s1569 -9056(12)60688-5.

35. Arlandis-Guzman S, Errando-Smet C, Trocio J, Arumi D, Rejas J. Cost-effectiveness analysis of antimuscarinics in the treatment of patients with overactive bladder in Spain: a decision-tree model. BMC Urol. 2011;11:9.

36. Irwin D, Milsom I, Reilly K, et al. Men and women with overactive bladder symptoms report higher prevalence of depression and lower quality of life: Results from the EPIC study. Neurourol Urodyn. 2006;25(6): abstract \#433.

37. Wielage RC, Perk S, Campbell NL, et al. Mirabegron for the treatment of overactive bladder: cost-effectiveness from US commercial health-plan and medicare advantage perspectives. J Med Econ. 2016;19(12):1135-43.

38. Herschorn S, Barkin J, Castro-Diaz D, et al. A phase III, randomized, double-blind, parallel-group, placebo-controlled, multicentre study to assess the efficacy and safety of the beta(3) adrenoceptor agonist, mirabegron, in patients with symptoms of overactive bladder. Urology. 2013;82(2):313-20.

39. Nitti VW, Auerbach S, Martin N, Calhoun A, Lee M, Herschorn $\mathrm{S}$. Results of a randomized phase III trial of mirabegron in patients with overactive bladder. J Urol. 2013;189(4):1388-95.

40. Yang Y, Brazier J, Tsuchiya A, Coyne K. Estimating a preference-based single index from the Overactive Bladder Questionnaire. Value Health J Int Soc Pharmacoecon Outcomes Res. 2009;12(1):159-66.

41. Desroziers K, Aballea S, Maman K, Nazir J, Odeyemi I, Hakimi Z. Estimating EQ-5D and OAB-5D health state utilities for patients with overactive bladder. Health Qual Life Outcomes. 2013;11:200

42. Nafees B, Stafford M, Gavriel S, Bhalla S, Watkins J. Health state utilities for non small cell lung cancer. Health Qual Life Outcomes. 2008;6:84.

43. Valenstein M, Vijan S, Zeber JE, Boehm K, Buttar A. The costutility of screening for depression in primary care. Ann Intern Med. 2001;134(5):345-60.

44. Lee BY, Bailey RR, Smith KJ, et al. Universal methicillin-resistant Staphylococcus aureus (MRSA) surveillance for adults at hospital admission: an economic model and analysis. Infect Control Hosp Epidemiol. 2010;31(6):598-606.

45. Djalalov S, Yong J, Beca J, et al. Genetic testing in combination with preventive donepezil treatment for patients with amnestic mild cognitive impairment: an exploratory economic evaluation of personalized medicine. Mol Diagn Ther. 2012;16(6):389-99.

46. Schädlich PK, Kentsch M, Weber M, et al. Cost Effectiveness of enoxaparin as prophylaxis against venous thromboembolic complications in acutely ill medical inpatients. PharmacoEconomics. 2006;24(6):571-91.

47. Instituto de Evaluación Tecnológica en Salud. Manual para la elaboración de evaluaciones económicas en salud. Bogotá: IETS; 2014.

48. Schem C, Bauerschlag DO, Gravert C, Heer IM, Jonat W, Strauss A. Economic aspects of abdominopelvic surgery in times of limited case-based compensation. Geburtshilfe und Frauenheilkunde. 2010;70(7):574-9.

49. Instituto de Seguros Sociales. Acuerdo 256 de 2001, 19 de diciembre, por el cual se aprueba el manual de tarifas de la entidad promotora de salud del Seguro Social EPS-ISS. Instituto de Seguros Sociales. 2001. https://lexsaludcolombia.files.wordpress. com/2010/10/tarifas-iss-2001.pdf. Accessed 11 Jan 2018.
50. Masís DP, Gómez-Restrepo C, Restrepo MU, et al. La carga económica de la depresión en Colombia: costos directos del manejo intrahospitalario. Revista Colombiana de Psiquiatría. 2010;39(3):465-80.

51. Sanchez J, Sanchez A, Cardona R. Economic consequences in real life of allergen immunotherapy in asthma, rhinitis and dermatitis. Revista alergia Mexico (Tecamachalco, Puebla, Mexico: 1993). 2016;63(4):323-33.

52. Olaya Acosta HA, Hernández Quintana MA. Infección de vías urinarias: Estudio costo-enfermedad y caracterización demográfica en una clínica de tercer nivel en Chía. Colombia: Universidad de La Sabana; 2012

53. Luster M, Felbinger R, Dietlein M, Reiners C. Thyroid hormone withdrawal in patients with differentiated thyroid Carcinoma: a one hundred thirty-patient pilot survey on consequences of hypothyroidism and a pharmacoeconomic comparison to recombinant thyrotropin administration. Thyroid. 2005;15(10):1147-55.

54. Anthony K, Ricotti V, Guglieri M, et al. Assessing T cell-mediated immune response to dystrophin in the natural history of Duchenne muscular dystrophy. J Neuromuscul Dis. 2014;1(Supplement 1):S128-9.

55. Philips Z, Bojke L, Sculpher M, Claxton K, Golder S. Good practice guidelines for decision-analytic modelling in health technology assessment: a review and consolidation of quality assessment. PharmacoEconomics. 2006;24(4):355-71.

56. Weinstein MC, O'Brien B, Hornberger J, et al. Principles of good practice for decision analytic modeling in health-care evaluation: report of the ISPOR Task Force on Good Research PracticesModeling Studies. Value Health J Int Soc Pharmacoecon Outcomes Res. 2003;6(1):9-17.

57. Kobelt G, Jonsson L, Mattiasson A. Cost-effectiveness of new treatments for overactive bladder: the example of tolterodine, a new muscarinic agent: a Markov model. Neurourol Urodyn. 1998;17(6):599-611.

58. Edwards SKC, Trevor N, Barton S, Nherera L. Mirabegron for the treatment of symptoms associated with overactive bladder. STA Report. London: BMJ-Technology Assessment Group; 2013.

59. Snedecor SJ. Review of economic value drivers of the treatment of overactive bladder. PharmacoEconomics. 2018;36(9):1083-92.

60. Fox C, Richardson K, Maidment ID, et al. Anticholinergic medication use and cognitive impairment in the older population: the medical research council cognitive function and ageing study. J Am Geriatr Soc. 2011;59(8):1477-83.

61. Fox C, Smith T, Maidment I, et al. Effect of medications with anti-cholinergic properties on cognitive function, delirium, physical function and mortality: a systematic review. Age Ageing. 2014;43(5):604-15.

62. Aging Brain Program of the Indiana University Center for Aging Research. Anticholinergic Cognitive Burden Scale 2012 Update. Indianapolis: Aging Brain Program of the Indiana University Center for Aging Research; 2012.

63. Panel AE. American Geriatrics Society updated Beers Criteria for potentially inappropriate medication use in older adults. J Am Geriatr Soc. 2012;60(4):616-31.

64. Stolk EA, Busschbach JJ. Validity and feasibility of the use of condition-specific outcome measures in economic evaluation. Qual Life Res Int J Qual Life Aspects Treat Care Rehabil. 2003;12(4):363-71.

65. Eurich DT, Johnson JA, Reid KJ, Spertus JA. Assessing responsiveness of generic and specific health related quality of life measures in heart failure. Health Qual Life Outcomes. 2006;4:89.

66. McKenna SP, Ratcliffe J, Meads DM, Brazier JE. Development and validation of a preference based measure derived from the Cambridge Pulmonary Hypertension Outcome Review (CAMPHOR) for use in cost utility analyses. Health Qual Life Outcomes. 2008;6:65. 